\title{
DISCOVERY OF TeV GAMMA-RAY EMISSION FROM CTA 1 BY VERITAS
}

\section{E. Aliu ${ }^{1}$, S. Archambault ${ }^{2}$, T. Arlen ${ }^{3}$, T. Aune ${ }^{4}$, M. Beilicke ${ }^{5}$, W. Benbow ${ }^{6}$, A. Bouvier $^{4}$, J. H. Buckley $^{5}$, V. Bugaev $^{5}$,} A. Cesarini ${ }^{7}$, L. Ciupik ${ }^{8}$, E. Collins-Hughes ${ }^{9}$, M. P. Connolly ${ }^{7}$, W. Cui ${ }^{10}$, R. Dickherber ${ }^{5}$, C. Duke $^{11}$, J. Dumm $^{12}$, V. V. Dwarkadas ${ }^{13}$, M. Errando ${ }^{1}$, A. Falcone ${ }^{14}$, S. Federici ${ }^{15,16}{ }^{\text {, Q. Feng }}{ }^{10}$, J. P. Finley ${ }^{10}$, G. Finnegan $^{17}$, L. Fortson $^{12}$, A. Furniss ${ }^{4}$, N. Galante ${ }^{6}$, D. Gall ${ }^{18}$, G. H. Gillanders ${ }^{7}$, S. Godambe $^{17}$, E. V. GotthelF ${ }^{19}$, S. GRiffin $^{2}$, J. Grube $^{8}$, G. GyuK ${ }^{8}$, D. Hanna ${ }^{2}$, J. Holder ${ }^{20}$, G. Hughes ${ }^{15}$, T. B. Humensky ${ }^{21}$, P. KAaret ${ }^{18}$, O. Kargaltsev ${ }^{22,23}$, N. Karlsson ${ }^{12}$, Y. KhASSEN ${ }^{9}$, D. KIEDA ${ }^{17}$, H. KRAWCZYNSKI ${ }^{5}$, F. KRENNRICH ${ }^{24}$, M. J. LANG ${ }^{7}$, K. LeE ${ }^{5}$, A. S. MADHAVAN ${ }^{24}$, G. MAIER ${ }^{15}$, P. Majumdar ${ }^{3}$, S. McArthur ${ }^{25}$, A. McCann ${ }^{26}$, P. Moriarty $^{27}$, R. Mukherjee ${ }^{1}$, T. Nelson ${ }^{12}$, A. O'Faoláin de Bhróithe 9 , R. A. Ong ${ }^{3}$, M. OrR ${ }^{24}$, A. N. Otte ${ }^{28}$, N. Park ${ }^{25}$, J. S. Perkins ${ }^{29,30}$, M. Pohl ${ }^{16,15}$, H. Prokoph ${ }^{15}$, J. QuinN $^{9}$, K. Ragan $^{2}$, L. C. Reyes ${ }^{31}$, P. T. Reynolds ${ }^{32}$, E. Roache ${ }^{6}$, M. Roberts ${ }^{33}$, D. B. SAXon ${ }^{20}$, M. Schroedter ${ }^{6}$, G. H. Sembroski ${ }^{10}$, P. Slane ${ }^{34}$, A. W. Smith ${ }^{17}$, D. StaszaK ${ }^{2}$, I. Telezhinsky ${ }^{15,16}$, G. TeŠIĆ ${ }^{2}$, M. Theiling $^{10}$, S. Thibadeau ${ }^{5}$, K. Tsurusaki $^{18}$, J. Tyler $^{2}$, A. Varlotta ${ }^{10}$, V. V. Vassiliev ${ }^{3}$, S. Vincent ${ }^{15}$, M. Vivier ${ }^{20}$, S. P. WaKely ${ }^{25}$, T. C. Weekes ${ }^{6}$, A. Weinstein $^{24}$, R. WELSING ${ }^{15}$, D. A. Williams ${ }^{4}$, AND B. ZITZER ${ }^{35}$

${ }^{1}$ Department of Physics and Astronomy, Barnard College, Columbia University, NY 10027, USA; muk@astro.columbia.edu

${ }^{2}$ Physics Department, McGill University, Montreal, QC H3A 2T8, Canada

${ }^{3}$ Department of Physics and Astronomy, University of California, Los Angeles, CA 90095, USA

${ }^{4}$ Santa Cruz Institute for Particle Physics and Department of Physics, University of California, Santa Cruz, CA 95064, USA ${ }^{5}$ Department of Physics, Washington University, St. Louis, MO 63130, USA

${ }^{6}$ Fred Lawrence Whipple Observatory, Harvard-Smithsonian Center for Astrophysics, Amado, AZ 85645, USA

${ }^{7}$ School of Physics, National University of Ireland Galway, University Road, Galway, Ireland

${ }^{8}$ Astronomy Department, Adler Planetarium and Astronomy Museum, Chicago, IL 60605, USA

${ }^{9}$ School of Physics, University College Dublin, Belfield, Dublin 4, Ireland

${ }^{10}$ Department of Physics, Purdue University, West Lafayette, IN 47907, USA

${ }^{11}$ Department of Physics, Grinnell College, Grinnell, IA 50112-1690, USA

${ }^{12}$ School of Physics and Astronomy, University of Minnesota, Minneapolis, MN 55455, USA

${ }^{13}$ Department of Astronomy and Astrophysics, University of Chicago, Chicago, IL 60637, USA

${ }^{14}$ Department of Astronomy and Astrophysics, 525 Davey Lab, Pennsylvania State University, University Park, PA 16802, USA

${ }^{15}$ DESY, Platanenallee 6, D-15738 Zeuthen, Germany

${ }^{16}$ Institute of Physics and Astronomy, University of Potsdam, D-14476 Potsdam-Golm, Germany

${ }^{17}$ Department of Physics and Astronomy, University of Utah, Salt Lake City, UT 84112, USA

18 Department of Physics and Astronomy, University of Iowa, Van Allen Hall, Iowa City, IA 52242, USA

${ }^{19}$ Columbia Astrophysics Laboratory, Columbia University, New York, NY 10027, USA

${ }^{20}$ Department of Physics and Astronomy and the Bartol Research Institute, University of Delaware, Newark, DE 19716, USA ${ }^{21}$ Physics Department, Columbia University, New York, NY 10027, USA

${ }^{22}$ Department of Astronomy, University of Florida, 205 Bryant Space Center, Gainesville, FL 32611, USA

${ }^{23}$ Department of Physics, The George Washington University, Washington, DC 20052, USA

${ }^{24}$ Department of Physics and Astronomy, Iowa State University, Ames, IA 50011, USA

25 Enrico Fermi Institute, University of Chicago, Chicago, IL 60637, USA; smcarthur@ulysses.uchicago.edu

${ }^{26}$ Kavli Institute for Cosmological Physics, University of Chicago, Chicago, IL 60637, USA
${ }^{27}$ Department of Life and Physical Sciences, Galway-Mayo Institute of Technology, Dublin Road, Galway, Ireland

${ }^{28}$ School of Physics and Center for Relativistic Astrophysics, Georgia Institute of Technology, 837 State Street NW, Atlanta, GA 30332-0430, USA

${ }^{29}$ CRESST and Astroparticle Physics Laboratory NASA/GSFC, Greenbelt, MD 20771, USA

${ }^{30}$ Department of Astronomy, University of Maryland, Baltimore County, 1000 Hilltop Circle, Baltimore, MD 21250, USA

${ }^{31}$ Physics Department, California Polytechnic State University, San Luis Obispo, CA 94307, USA

32 Department of Applied Physics and Instrumentation, Cork Institute of Technology, Bishopstown, Cork, Ireland

${ }^{33}$ Eureka Scientific, Inc., Oakland, CA 94602, USA

${ }^{34}$ Harvard-Smithsonian Center for Astrophysics, 60 Garden Street, Cambridge, MA 02138, USA

${ }^{35}$ Argonne National Laboratory, 9700 S. Cass Avenue, Argonne, IL 60439, USA

Received 2012 September 4; accepted 2012 December 11; published 2013 January 23

\section{ABSTRACT}

We report the discovery of $\mathrm{TeV}$ gamma-ray emission coincident with the shell-type radio supernova remnant (SNR) CTA 1 using the VERITAS gamma-ray observatory. The source, VER J0006+729, was detected as a 6.5 standard deviation excess over background and shows an extended morphology, approximated by a two-dimensional Gaussian of semimajor (semiminor) axis $0.30(0.24)$ and a centroid $5^{\prime}$ from the Fermi gamma-ray pulsar PSR J0007+7303 and its X-ray pulsar wind nebula (PWN). The photon spectrum is well described by a power-law $d N / d E=N_{0}(E / 3 \mathrm{TeV})^{-\Gamma}$, with a differential spectral index of $\Gamma=2.2 \pm 0.2_{\text {stat }} \pm 0.3_{\text {sys }}$, and normalization $N_{0}=\left(9.1 \pm 1.3_{\text {stat }} \pm 1.7_{\text {sys }}\right) \times 10^{-14} \mathrm{~cm}^{-2} \mathrm{~s}^{-1} \mathrm{TeV}^{-1}$. The integral flux, $F_{\gamma}=4.0 \times 10^{-12} \mathrm{erg} \mathrm{cm}^{-2} \mathrm{~s}^{-1}$ above $1 \mathrm{TeV}$, corresponds to $0.2 \%$ of the pulsar spin-down power at $1.4 \mathrm{kpc}$. The energetics, colocation with the SNR, and the relatively small extent of the $\mathrm{TeV}$ emission strongly argue for the PWN origin of the $\mathrm{TeV}$ photons. We consider the origin of the $\mathrm{TeV}$ emission in CTA 1.

Key words: gamma-rays: stars - pulsars: individual (PSR J0007+7303) - supernovae: individual (G119.5+10.2) X-rays: individual (RX J0007.0+7303)

Online-only material: color figures 


\section{INTRODUCTION}

There are many possible associations of gamma-ray sources with supernova remnants (SNRs). These gamma rays could come from shock acceleration in the shell, a pulsar associated with the SNR, or a pulsar wind nebula (PWN) surrounding the pulsar. Such gamma-ray/SNR associations date back to $C O S B$ observations of SNRs coincident with OB stellar associations (Montmerle 1979). Observations of the Galaxy by EGRET in the energy range $30 \mathrm{MeV}-30 \mathrm{GeV}$ revealed $\sim 19$ unidentified sources at low Galactic latitudes that were found to be spatially correlated with mostly shell-type SNRs (Torres et al. 2003). One such source was 3EG J0010+7309, with a relatively small $95 \%$ error circle of $28^{\prime}$ (Hartman et al. 1999), that was spatially coincident with the SNR CTA $1($ G119.5+10.2) and the X-ray point source RX J0007.0+7303, which was postulated to be a pulsar (Brazier et al. 1998). The association between 3EG J0010+7309 and RX J0007.0+7303 was found to be plausible, given the lack of flux variability seen in 3EG J0010+7309, its hard spectral index $(\Gamma=1.58 \pm 0.18$ between $70 \mathrm{MeV}$ and $2 \mathrm{GeV}$ ), and its similarity with other known pulsars detected by EGRET (Brazier et al. 1998).

CTA 1 is a composite SNR, discovered by Harris \& Roberts (1960), with a shell-type structure in the radio band and a center-filled morphology at X-ray energies. The radio shell is incomplete toward the northwest (NW) of the remnant, possibly due to rapid expansion of the shock into a lower-density region (Pineault et al. 1993). The distance to SNR CTA 1 as derived from the associated $\mathrm{H}$ i shell is $d=1.4 \pm 0.3 \mathrm{kpc}$ (Pineault et al. 1997), the SNR age is estimated to be $\sim 1.3 \times 10^{4}$ yr (Slane et al. 2004), and the diameter of its radio shell is $\sim 1.8$ (Sieber et al. 1981).

Archival X-ray observations of SNR CTA 1 in the 5-10 keV band show non-thermal diffuse emission of low surface brightness in the center of the remnant, likely corresponding to a PWN driven by an active neutron star (Slane et al. 1997). The neutron star candidate RX J0007.0+7303 is a faint source located at the brightest part of the synchrotron emission (Seward et al. 1995). A Chandra image of RX J0007.0+7303 provides further evidence that this source is an energetic rotation-powered pulsar, resolving a central point source, a compact nebula, and a bent jet (Halpern et al. 2004). An initial observation with XMM-Newton in 2002 found the X-ray spectrum of the central source to be consistent with that of a neutron star, although no pulsations were detected (Slane et al. 2004). Based on these initial X-ray observations, the spin-down luminosity of the underlying pulsar was estimated to be in the range $10^{36}-10^{37} \mathrm{erg} \mathrm{s}^{-1}$, supporting the identification of the EGRET source 3EG J0010+7309 as a pulsar (Slane et al. 2004; Halpern et al. 2004).

Eventually, a search for pulsed GeV emission from CTA 1 using the data from the Fermi Gamma-Ray Space Telescope revealed a highly significant $316 \mathrm{~ms}$ signal, confirming the origin of 3EG J0010+7309 (Abdo et al. 2008). The spin-down power was determined to be $\sim 4.5 \times 10^{35} \mathrm{erg} \mathrm{s}^{-1}$, which is sufficient to power the PWN (Slane et al. 2004). Following the Fermi discovery of the gamma-ray pulsar, a deep $130 \mathrm{ks}$ observation of RX J0007.0+7303 was carried out with XMMNewton to characterize the timing behavior. The X-ray signal of PSR J0007+7303 was discovered at a statistical significance of $4.7 \sigma$ in the $0.5-2 \mathrm{keV}$ band, out of phase with the gamma-ray pulse (Caraveo et al. 2010). Similar to Geminga (Halpern \& Holt 1992) and PSR J1836+5925 (Halpern et al. 2007; Abdo et al. 2010), PSR J0007+7303 is also radio quiet. GeV emission in the off-pulse phase interval has also recently been detected by Fermi (Abdo et al. 2012).

Many galactic $\mathrm{TeV}$ sources appear to be associated with pulsars via their wind nebulae, comprised of relativistic wind particles confined by the pressure of the surrounding medium (Gaensler \& Slane 2006). The initially highly supersonic wind terminates in a shock that can be associated with axisymmetric, toroidal structures often seen in the X-ray images of PWNe (e.g., Kargaltsev \& Pavlov 2008). PWNe now represent the most populous class of Galactic TeV emitters (Hinton \& Hofmann 2010). The non-thermal emission seen in PWNe from the radio up to gamma rays below a few $\mathrm{GeV}$ or less is generally interpreted as synchrotron radiation from the accelerated leptons. The emission observed at higher energies, up to several $\mathrm{TeV}$, can be produced via inverse Compton (IC) scattering of these same high-energy electrons with background photons (e.g., the cosmic microwave background (CMB), infrared radiation from dust, starlight, and synchrotron photons; Atoyan \& Aharonian 1996). Alternatively, hadronic mechanisms may also be responsible for the $\mathrm{TeV}$ emission, in which case the wind should be composed of relativistic hadrons that collide with the ambient medium and produce pions, with the $\mathrm{TeV}$ emission coming from $\pi^{0}$ decay. To date, however, there has been no solid evidence requiring a large contribution to the gamma-ray emission from such hadronic processes.

PWNe experience several stages of evolution (e.g., Gaensler $\&$ Slane 2006). At an early stage, the pulsar wind freely expands into the SN ejecta. For a slowly moving pulsar, the PWN is approximately centered on the pulsar, while for a supersonically moving pulsar the PWN will take a cometary shape. At later times, the PWN is compressed by the reverse SNR shock and may be displaced significantly from the pulsar if the reverse shock is asymmetric. Such crushed and displaced PWNe have been dubbed relic PWNe.

The X-ray and gamma-ray observations of CTA 1 suggest that the extended non-thermal emission around the gammaray pulsar is a synchrotron PWN. Motivated by these observations, model calculations by Zhang et al. (2009) suggested that the $\mathrm{TeV}$ emission is largely produced by the PWN and that the level of emission should be detectable at TeV energies by VERITAS. For IC scattering off the PWN electrons, Zhang et al. (2009) predicted a gamma-ray flux $F_{\gamma}(1-30 \mathrm{TeV}) \sim$ $1.1 \times 10^{-12} \mathrm{erg} \mathrm{cm}^{-2} \mathrm{~s}^{-1}$. Previous TeV observations of CTA 1 by the earlier imaging atmospheric Cherenkov telescopes gave upper limits, as follows: $2.64 \times 10^{-11}$ photons $\mathrm{cm}^{-2} \mathrm{~s}^{-1}$ $(E>250 \mathrm{GeV})$ by CAT (Khelifi et al. 2001), $1.25 \times$ $10^{-11}$ photons $\mathrm{cm}^{-2} \mathrm{~s}^{-1}(E>620 \mathrm{GeV})$ by Whipple (Hall et al. 2001), and $1.09 \times 10^{-12}$ photons $\mathrm{cm}^{-2} \mathrm{~s}^{-1}(E>1.3 \mathrm{TeV})$ by HEGRA (Rowell et al. 2003).

In this paper, we report the Very Energetic Radiation Imaging Telescope Array System (VERITAS) detection of TeV emission from the central region of CTA 1 .

\section{VERITAS INSTRUMENT AND OBSERVATIONS}

The VERITAS uses ground-based detection techniques pioneered by its predecessor, the Whipple $10 \mathrm{~m}$ Telescope (Weekes et al. 1989), to explore the universe in very high energy (VHE) gamma rays from $\sim 100 \mathrm{GeV}$ to $\sim 30 \mathrm{TeV}$. The VERITAS telescope array consists of four $12 \mathrm{~m}$ diameter Davies-Cotton telescopes and is located at the base camp of the Fred Lawrence Whipple Observatory in southern Arizona (Holder et al. 2011). Flashes of Cherenkov light from gamma-ray and cosmic-ray 
showers are focused by a set of mirrors onto a camera located in the focal plane of each telescope. Each camera comprises 499 photomultiplier tube pixels and light concentrators arranged in a hexagonal pattern with a total field of view of 3.5. Stereoscopic imaging of showers from multiple viewing angles allows the determination of the shower core location relative to the array using simple geometric reconstruction techniques that rely on the fact that the major axes of the shower images are projections of the shower axis. The combined instrument has an angular resolution of $<0.1$ ( $68 \%$ containment) and energy resolution of $15 \%-20 \%$ for energies $>200 \mathrm{GeV}$. It can detect a source with a flux of $1 \%$ of the steady Crab Nebula VHE flux at a 5 standard deviation significance level in less than $30 \mathrm{hr}$ (Ong et al. 2009).

A three-level trigger system is used to help eliminate background noise. The first trigger occurs at the pixel level, requiring the signal to reach a $50 \mathrm{mV}$ threshold (corresponding to 4-5 photoelectrons) set by a constant fraction discriminator (CFD). The second, telescope-level trigger requires at least three adjacent pixels passing the CFD trigger to form an image. A third, array-level trigger requires simultaneous Cherenkov images in at least two telescopes, within a $50 \mathrm{~ns}$ time window, which then causes a readout of the $500 \mathrm{MSample} / \mathrm{s}$ Flash-ADC data acquisition system for each pixel.

CTA 1 was observed over two epochs. The first set of observations spanned from 2010 September to 2011 January, with a total livetime of $25 \mathrm{hr} 39$ minutes after data-quality selection based on weather conditions and hardware status. An additional $15 \mathrm{hr} 36$ minutes of quality-selected data were taken from 2011 September to December. Observations were taken in "wobble" mode (Fomin et al. 1994), in which the telescope pointing is offset from the source position by some angular distance. This method allows for simultaneous collection of data and estimation of the background. Due to the extended nature of the remnant and expected extension of the PWN, an offset distance of 0.7 was used, larger than the typical VERITAS distance of 0.5 . To decrease bias, the offset direction was varied between each 20 minute run while maintaining the same offset distance, alternating between the north, south, east, and west directions (in the equatorial coordinate system). Observations were taken in a narrow range of zenith angles, $40^{\circ}-47^{\circ}$, with an average of 42.5 for the full data set. All of the data presented here were taken with all four telescopes in the array.

\section{ANALYSIS}

The CTA 1 data were processed using standard VERITAS analysis techniques, as described in Acciari et al. (2008). The cosmic-ray background was suppressed efficiently by parameterizing the recorded shower images by their principal moments (Hillas 1985), and the shower direction and impact parameter were reconstructed from these images, using stereoscopic methods (see, e.g., Aharonian et al. 1997; Krawczynski et al. 2006). Gamma-ray/hadronic shower separation is achieved through selection criteria (cuts). Based upon the predicted spectrum of Zhang et al. (2009), two sets of standard cuts were used. These cuts were optimized on simulations for sources of $\sim 5 \%$ of the Crab Nebula flux and with moderate and hard spectral indices $(\sim-2.5$ and -2.0 , respectively). For these cuts, at least three of the telescopes in the array had to have images recorded in the camera, with at least 1200 digital counts $(\sim 240$ photoelectrons) for the hard-cut analysis and 500 digital counts ( $\sim 95$ photoelectrons) for the moderate-cut analysis. Cuts were also applied to the mean scaled length (MSL), mean scaled width (MSW), and integrated charge in the signal (size). Finally

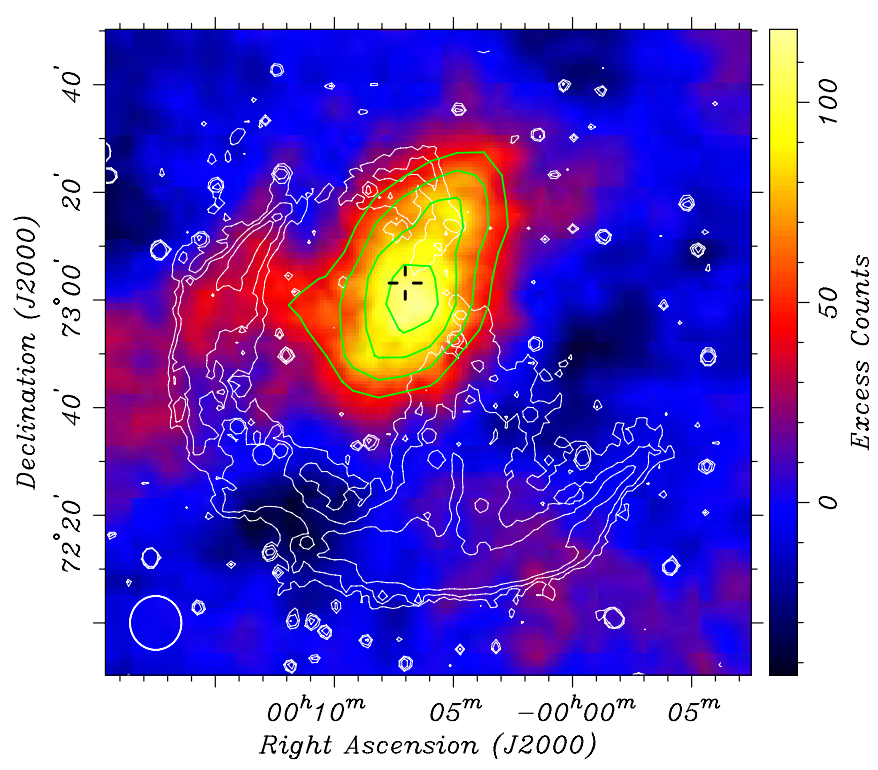

Figure 1. VERITAS excess map of the region around SNR CTA 1 using a hardspectrum analysis. The color scale indicates excess gamma-ray events within an integration radius of 0.23 . The circle at the lower left corner shows the size of the point-spread function ( $68 \%$ containment). The radio contours at $1420 \mathrm{MHz}$ (Pineault et al. 1997) are overlaid in white, showing the SNR shell. The VERITAS significance contours at $3 \sigma, 4 \sigma, 5 \sigma$, and $6 \sigma$ are shown in green. The cross marks the position of the pulsar (Abdo et al. 2008), located $5^{\prime} \pm 2^{\prime}$ from the centroid of $\mathrm{TeV}$ emission. North is up and east is to the left.

(A color version of this figure is available in the online journal.)

a cut was applied on $\theta$, the angular distance in the field of view from the reconstructed arrival direction of the event to the putative source location. A cut of $\theta<0.09(\theta<0.23)$ was used for a point-source (extended-source) search, with the size of the extended-source cut selected a priori. For the analysis presented here, the cuts for the moderate- and hard-spectra analysis are MSW $<0.35$ and MSL $<0.7$.

The background was estimated using the ring background model (e.g., see Berge et al. 2007), with a ring of mean radius 0.7 and a background to source area ratio of 8.0. Regions in the field of view containing stars of $B$ magnitude brighter than 6.0 were excluded from the background estimation in order to reduce systematic errors. The statistical significance of the excess was calculated using Equation 17 of $\mathrm{Li} \& \mathrm{Ma}$ (1983). The energy threshold for this analysis after applying the moderate (hard) cuts is $\sim 550 \mathrm{GeV}(1 \mathrm{TeV})$ at a zenith angle of $45^{\circ}$, with a systematic error of about $20 \%$ on the energy estimation. Two independent analysis packages, as described by Cogan (2008) and Daniel (2008), were used to reproduce the results presented here on CTA 1.

\section{RESULTS}

Figure 1 shows the $\mathrm{TeV}$ excess map of the region of the sky around CTA 1. The hard-spectrum, extended-source analysis produced an excess with a significance of 7.5 standard deviation $(\sigma)$ pre-trials, in a search region of radius 0.4 around the pulsar PSR J0007+7303, within the radio shell of the SNR CTA 1. Accounting for the two sets of applied cuts with two different integration radii, and determining the a priori trials factor by tiling the search region with 0.04 bins (Aharonian et al. 2006), we estimate the post-trials significance of detection to be $6.5 \sigma$. Overlaid on the $\mathrm{TeV}$ image are the high-resolution radio contours at $1420 \mathrm{MHz}$, obtained using the Dominion 


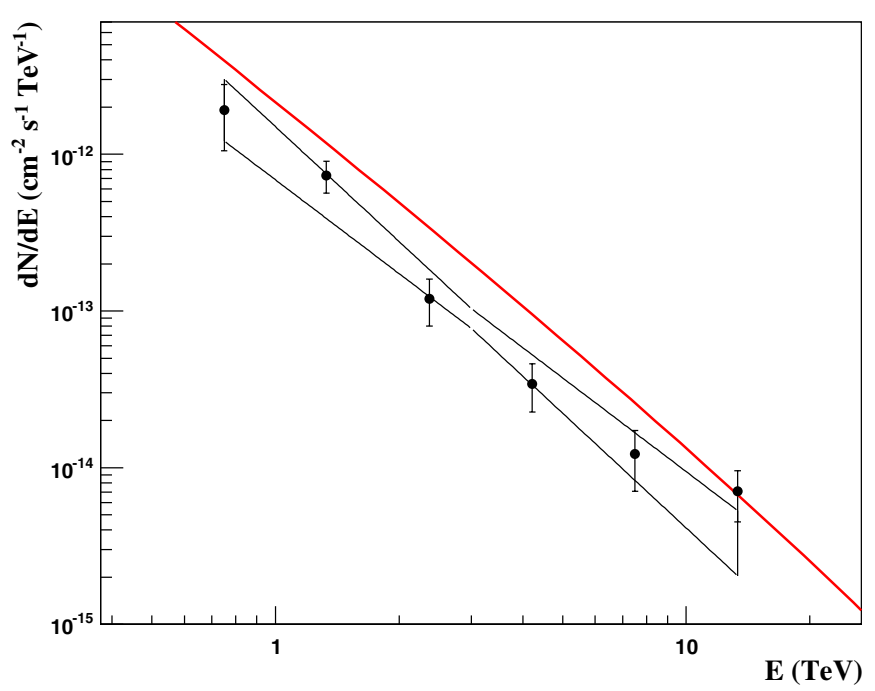

Figure 2. VERITAS differential gamma-ray spectrum of CTA 1. The black butterfly shows the uncertainties of the best-fit power-law model. The red line marks the flux predicted by Zhang et al. (2009, Figure 4). The errors are statistical only.

(A color version of this figure is available in the online journal.)

Table 1

Differential Flux Measurements of CTA 1 with VERITAS. The Errors are Statistical Only

\begin{tabular}{lcc}
\hline \hline $\begin{array}{l}\text { Energy Range } \\
(\mathrm{TeV})\end{array}$ & $\begin{array}{l}\text { Flux } \\
\left(\mathrm{cm}^{-2} \mathrm{~s}^{-1} \mathrm{TeV}^{-1}\right)\end{array}$ & $\begin{array}{c}\text { Significance } \\
(\sigma)\end{array}$ \\
\hline $0.56-1.00$ & $(1.9 \pm 0.9) \times 10^{-12}$ & 2.3 \\
$1.00-1.78$ & $(7.3 \pm 1.7) \times 10^{-13}$ & 4.5 \\
$1.78-3.16$ & $(1.2 \pm 0.4) \times 10^{-13}$ & 3.3 \\
$3.16-5.62$ & $(3.4 \pm 1.2) \times 10^{-14}$ & 3.1 \\
$5.62-10.00$ & $(1.2 \pm 0.5) \times 10^{-14}$ & 2.5 \\
$10.00-17.78$ & $(7.1 \pm 2.5) \times 10^{-15}$ & 2.8 \\
\hline
\end{tabular}

Radio Astrophysical Observatory Synthesis Telescope, and the Effelsberg $100 \mathrm{~m}$ telescope, showing bright radio arcs visible to the south and east, with an incomplete shell in the northwest, possibly due to the breakout of the SNR blast wave into a medium of lower density (Pineault et al. 1993, 1997).

For spectral analysis, the moderate-spectrum cuts were used in order to provide the lowest energy threshold for the analysis. The differential photon spectrum above $500 \mathrm{GeV}$ is shown in Figure 2, with spectral data points listed in Table 1. The spectrum is generated with the reflected-region background model (Berge et al. 2007) with $41 \mathrm{hr} 15$ minutes of quality-selected data. The spectrum can be fit with a power law of the form $d N / d E=$ $N_{0}(E / 3 \mathrm{TeV})^{-\Gamma}$, with $\Gamma=2.2 \pm 0.2_{\text {stat }} \pm 0.3_{\text {sys }}$ and $N_{0}=$ $\left(9.1 \pm 1.3_{\text {stat }} \pm 1.7_{\text {sys }}\right) \times 10^{-14} \mathrm{~cm}^{-2} \mathrm{~s}^{-1} \mathrm{TeV}^{-1}$. The integral energy flux above $1 \mathrm{TeV}, F_{\gamma}=4.0 \times 10^{-12} \mathrm{erg} \mathrm{cm}^{-2} \mathrm{~s}^{-1}$, corresponds to $0.2 \%$ of the pulsar spin-down luminosity at $1.4 \mathrm{kpc}$ and $\sim 4 \%$ of the steady $\mathrm{TeV}$ gamma-ray emission from the Crab Nebula.

\subsection{Morphology}

Figure 1 shows that the extent of the $\mathrm{TeV}$ gamma-ray emission region in CTA 1 exceeds the point-spread function (PSF; $68 \%$ containment radius of the events coming from a point source) of VERITAS. In order to estimate the extent of the source, an asymmetric two-dimensional Gaussian is fit to the acceptance-corrected uncorrelated map of excess events binned in 0.05 bins. Although the shape and extent of the emission

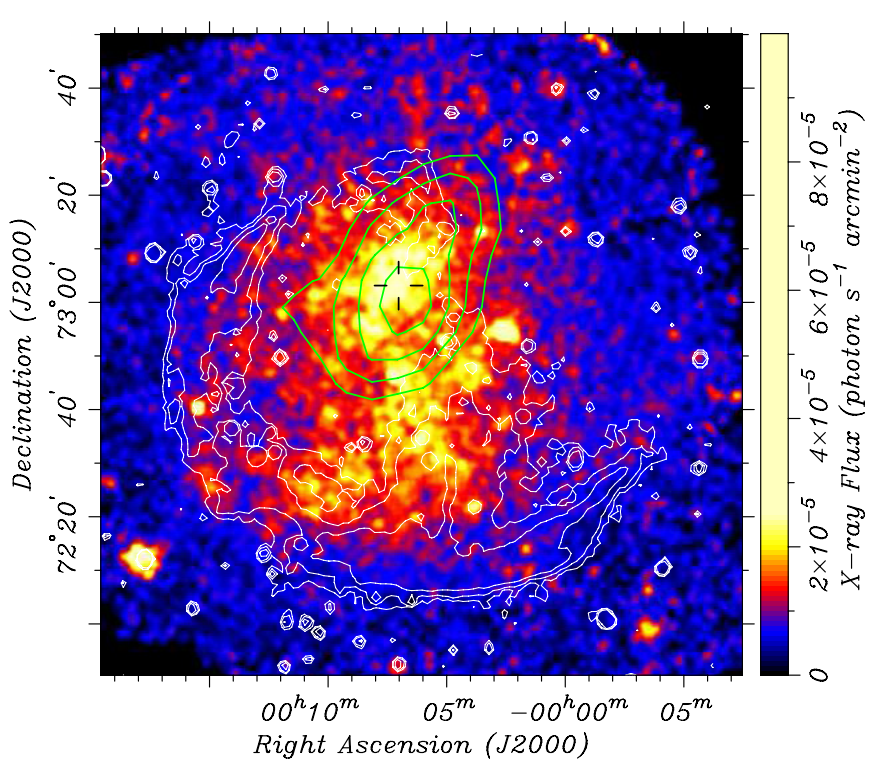

Figure 3. ROSAT X-ray image $(0.5-2.0 \mathrm{keV})$ of the SNR CTA 1 shown in equatorial coordinates. The cross marks the location of the X-ray point source RX J0007.0+7303 and the Fermi PSR J0007+7303. The SNR shell is shown by the $1420 \mathrm{MHz}$ radio contours (Pineault et al. 1997), overlaid in white. The VERITAS significance contours at $3 \sigma, 4 \sigma, 5 \sigma$, and $6 \sigma$ are shown in green.

(A color version of this figure is available in the online journal.)

is likely more complex than a simple asymmetric Gaussian, as a first approximation, it still provides a statistically reasonable estimate of the source extent. Due to the finite resolution of the detector, the emission we see is a convolution of the real source and the PSF describing the system. Accounting for the PSF of the instrument, the resulting $1 \sigma$ angular extent is $0.30 \pm 0.03$ along the semimajor axis and $0.24 \pm 0.03$ along the semiminor axis, with an orientation angle of $17.4 \pm 15.8$ west of north. We note that this is a sensitivity-limited measurement.

The fitted centroid location is $00^{\mathrm{h}} 06^{\mathrm{m}} 26^{\mathrm{s}}$, $+72^{\circ} 59^{\prime} 01^{\prime \prime} .0$ (J2000), which is 5 arcmin from PSR J0007+7303. Therefore, the VERITAS source name is VER J0006+729. The statistical uncertainty in the centroid position is 0.09 in R.A. and 0.04 in declination. The systematic uncertainty in the position due to the telescope pointing error is $50^{\prime \prime}$.

\subsection{Archival X-Ray Analysis}

Figure 3 shows the exposure-corrected, smoothed ROSAT PSPC X-ray image of the region around CTA 1 . The $0.5-2 \mathrm{keV}$ ROSAT image reveals a center-filled morphology, with a faint compact source located at the peak of the brightness distribution. The cross in the image marks the location of the X-ray point source RX J0007.0+7303 and the Fermi pulsar J0007+7303. The pulsar is located close to the center of the extended $\mathrm{TeV}$ source with $\sim 5^{\prime}$ offset from the peak of the $\mathrm{TeV}$ surface brightness. Figure 4 shows the non-thermal X-ray image from $A S C A$ in the 4-10 keV band (Roberts et al. 2001), along with the $\mathrm{TeV}$ contours. While the non-thermal emission and the $\mathrm{TeV}$ emission are both clearly extended with centroids separated by only 5 arcmin, the limited angular resolution and signal-to-noise ratio $(\mathrm{S} / \mathrm{N})$ in the VERITAS data do not allow for a more rigorous comparison.

Figure 5 is the smoothed XMM-Newton image of the vicinity of PSR J0007+7303 (ObsID: 0604940101, PI P. Caraveo) showing the X-ray PWN. The inset shows the smoothed Chandra image (ObsID: 3835, PI J. Halpern) revealing a 


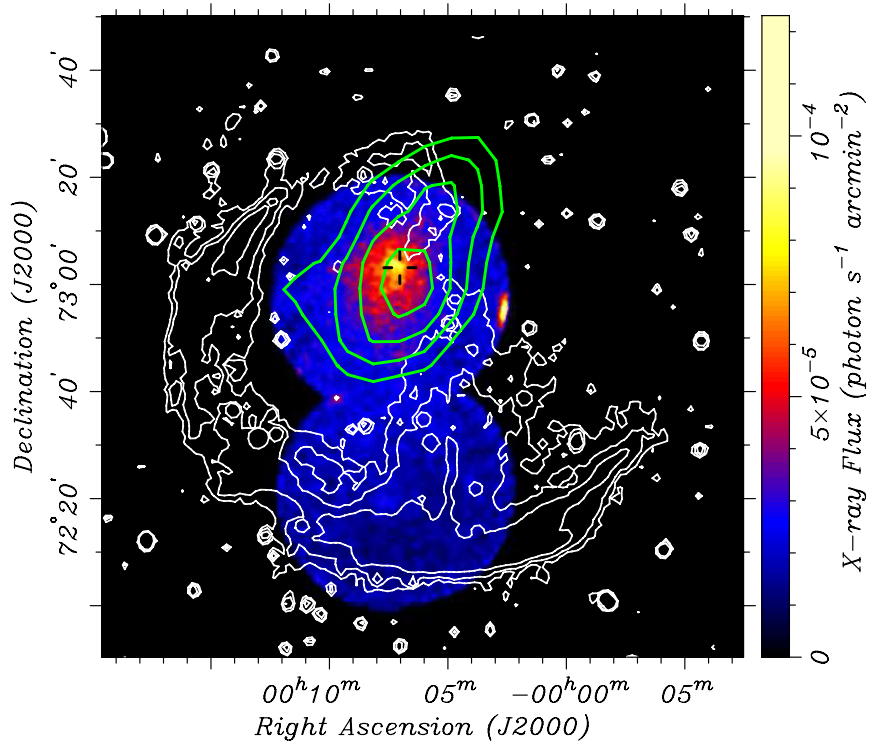

Figure 4. ASCA GIS image (4-10 keV) of the SNR CTA 1, using the same field of view as Figures 1 and 3. The position of PSR J0007+7303 is marked by the cross. The $1420 \mathrm{MHz}$ radio contours are shown in white. The VERITAS significance contours at $3 \sigma, 4 \sigma, 5 \sigma$, and $6 \sigma$ are shown in green.

(A color version of this figure is available in the online journal.)

compact nebula and bent jet attached to the point source, along with diffuse emission at larger scales. The Chandra jet is particularly apparent in the analysis presented by Halpern et al. (2004), where it is estimated that the Chandra point source accounts for $\sim 30 \%$ of the flux of RX J0007.0+7303, with the compact nebula plus jet comprising the remaining $\sim 70 \%$. The luminosity of the fainter large-scale emission (within $r<4^{\prime}$ from the pulsar) is about a factor of 5-10 larger than that of the compact PWN and pulsar. The X-ray spectrum of the point source can be described by an absorbed power law plus blackbody model, with a photon index of $\Gamma=1.6 \pm 0.6$. The compact PWN spectrum is harder, with a photon index $\Gamma \simeq 1-1.3$ (Halpern et al. 2004).
The spectrum of the large-scale diffuse emission was fitted by Caraveo et al. (2010) with a power law modified by the interstellar absorption. The fit gave $\Gamma=1.8 \pm 0.1$. However, the fit quality was fairly poor suggesting a more complex spectrum (e.g., a possible additional thermal component; see below).

\section{DISCUSSION}

We have discovered spatially extended $\mathrm{TeV}$ emission from the region of CTA 1 . Here, we discuss the results in the context of the available multiwavelength data.

\subsection{The Nature of the TeV Source: A PWN Scenario}

The good positional match between VER J0006+729 and PSR J0007+7303 within the CTA 1 SNR makes their physical association virtually indisputable, while leaving uncertain which component powers the $\mathrm{TeV}$ emission. The source is unlikely to be related to a gamma-ray binary or a background blazar given that the $\mathrm{TeV}$ emission is extended and non-variable. However, the extent of VER J0006+729 is also much smaller than that of the SNR and, hence, the TeV source does not resemble several SNRs whose shells have been resolved in TeV gamma rays (Komin et al. 2011). There still remains a possibility that only part of the CTA 1 SNR shell is interacting with a dense molecular cloud which could cause a local enhancement in the $\mathrm{TeV}$ brightness of the emission (Komin et al. 2011). However, we do not find any evidence for such a cloud at any other wavelength, including $60 \mu \mathrm{m}$ IR or H I (see Pineault et al. 1993), and the high Galactic latitude of the SNR places it nearly $250 \mathrm{pc}$ above the Galactic plane, much higher than the scale height of molecular clouds.

VER J0006+729 must then be powered by the young PSR J0007+7303. Extrapolating the pulsed emission seen by the Fermi-LAT (Abdo et al. 2012), assuming a power law above the break energy of $\sim 4 \mathrm{GeV}$, gives an estimated $\mathrm{TeV}$ flux 2-3 orders of magnitude below the observed flux. Therefore, the most plausible remaining scenario is that the $\mathrm{TeV}$ emission originates from the X-ray PWN surrounding the pulsar. The

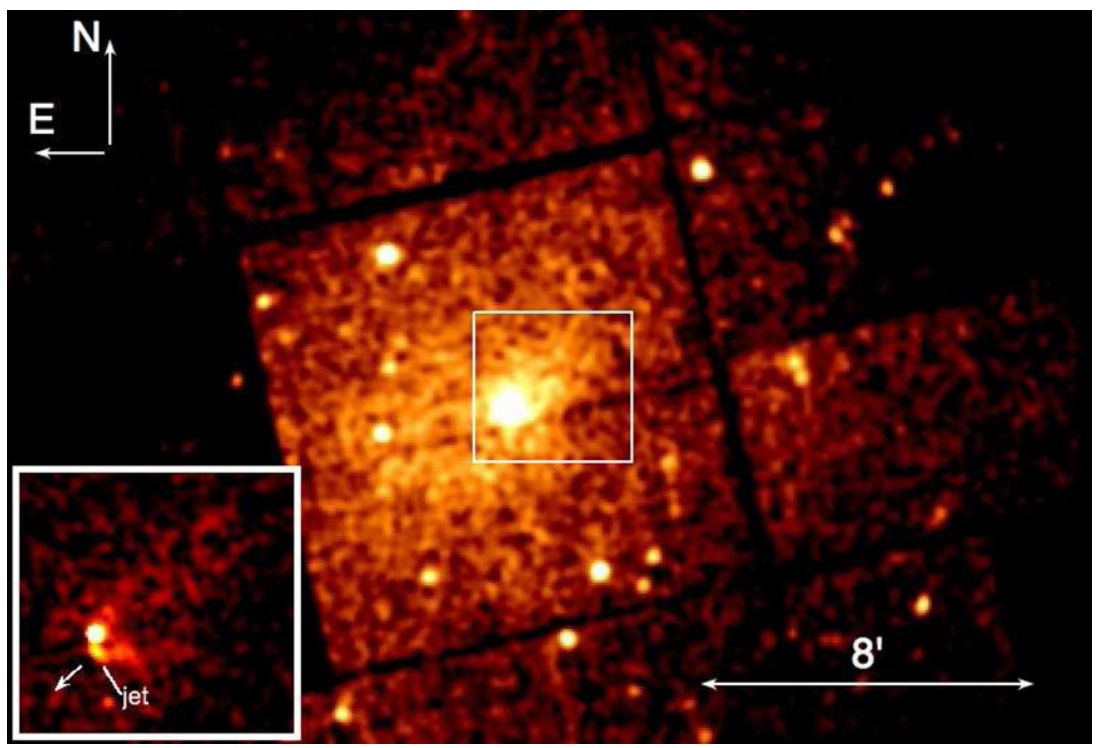

Figure 5. XMM-Newton $93 \mathrm{ks}$ EPIC/MOS1+2 image ( $0.5-10 \mathrm{keV}$; pixel size $4^{\prime \prime}$; smoothed with the $r=12^{\prime \prime}$ Gaussian kernel) of PSR J0007+7303 and its vicinity showing the X-ray PWN. The inset is the higher resolution $50 \mathrm{ks}$ Chandra ACIS image (0.5-8 keV; pixel size 2", smoothed with the $r=4^{\prime \prime}$ Gaussian kernel). The arrow in the inset image shows the likely direction of the pulsar proper motion (based on the shape of the compact PWN).

(A color version of this figure is available in the online journal.) 
PWN consists of a bent jet, a compact core, and a large-scale diffuse component, as seen in the Chandra and XMM-Newton images (see Figure 5). The bending of the jet (see the inset in Figure 5, and also Halpern et al. 2004) could be caused by the ram pressure of the oncoming medium due to the NW-SE pulsar motion or by the interaction with a reverse shock propagating NW within the SNR extent. Alternatively, a kink instability might be responsible for the bending of the jet. Indeed, the Vela pulsar jet shows some kink-like shape changes. However, these wiggles tend to occur on smaller spatial scales, while globally the Vela jet is always (during the last 10 years) seen to bend toward one side, likely due to the pressure of the oncoming ambient material (Pavlov et al. 2003). Similarly, in the XMMNewton and Chandra images of CTA 1, obtained at different epochs, the jet is seen to consistently bend in the same direction.

If the change in the jet morphology is caused by ram pressure, then we can estimate the pressure from the jet's curvature, following Pavlov et al. (2003). Assuming that the jet pressure is dominated by the contribution from a magnetic field $B_{-4}=B /\left(10^{-4} \mathrm{G}\right)$, for a jet curvature radius $R_{\text {curv }} \simeq$ $10^{\prime \prime}$ and a jet diameter $d_{\text {jet }} \sim 1^{\prime \prime}$ the pressure estimate is $P_{\text {ram }} \sim 1 \times 10^{-11} B_{-4}^{2}$ erg $\mathrm{cm}^{-3}$. Despite the fairly high magnetic field assumed, the estimated pressure is rather low compared to the ambient pressure inferred for other young pulsars with X-ray PWNe resolved by Chandra (Kargaltsev \& Pavlov 2008). Assuming that the ram pressure is caused by the pulsar motion through a medium of density $n_{-1}=$ $n /\left(10^{-1} \mathrm{~cm}^{-3}\right)$, one obtains a very modest pulsar speed $v \simeq$ $90 n_{-1}^{-1 / 2} B_{-4} \mathrm{~km} \mathrm{~s}^{-1}$ which corresponds to the proper motion of just $\sim 0.013 n_{-1}^{-1 / 2} B_{-4} d_{1.4}^{-1} \operatorname{arcs} \mathrm{yr}^{-1}$, assuming a distance of $d_{1.4}=d /(1.4 \mathrm{kpc})$. This means that over its lifetime $\tau$ the pulsar should have moved by only $\sim 3 n_{-1}^{-1 / 2}(\tau / 1.3 \times$ $\left.10^{4} \mathrm{yr}\right) B_{-4} d_{1.4}^{-1}$ arcmin. This distance is much less than the size of the SNR and the extent of the TeV source, and it is even less than the extent of large-scale X-ray PWN seen in the XMM-Newton and $A S C A$ images. We also note that despite being dependent on several parameters, the above estimate of the distance traveled by the pulsar likely represents an upper limit. Thus, the estimated velocity from the jet-bending is inconsistent with the otherwise plausible hypothesis that the NW extension of the TeV source (see Figure 1) might be due to aged relativistic electrons left behind by the fast moving pulsar.

It is also possible that the relic PWN has been pushed to one side (i.e., NW of the pulsar) by the reverse shock that must have arrived from the SE direction. Indeed, such a scenario is supported by the overall asymmetry of the SNR shell which appears to expand into much lower density medium in its NW part and hence the reverse shock is not expected to arrive from the NW direction. A recent interaction with the reverse shock could possibly also explain the bending of the jet while emission ahead of the pulsar could be explained by the turbulent mixing between the pulsar wind and SN ejecta behind the reverse shock (similar to G327.1-1.1; Temim et al. 2009). The latter may contribute some thermal emission and explain the poor quality of the power-law fit to the extended X-ray emission (Caraveo et al. 2010). A deep mapping of the entire SNR by XMM-Newton can test this hypothesis by providing a high $\mathrm{S} / \mathrm{N}$ spectrum of the faint large-scale X-ray emission which should then contain a thermal emission component coming from the ejecta (cf., e.g., Vela X spectrum; LaMassa et al. 2008).

Pulsar wind particles may be transported either by diffusion or by advection. One could in principle compare the two terms if the bulk flow speed (as a function of distance from the pulsar) and the magnetic field structure were known. The MHD models for isotropic pulsar winds (e.g., Kennel \& Coroniti 1984) are unlikely to be valid on large scales and when mixing due to interaction with the reverse shock is present. However, we can make some estimates of the average magnetic field by assuming which process is dominant in transporting the particles.

Assuming that X-ray- and TeV-emitting particles move away from the pulsar with similar velocities (i.e., that the X-ray- and TeV-emitting regions are cospatial and that the effects of energydependent diffusion are negligible), and that the synchrotron cooling time is the dominant timescale, one can crudely estimate the magnetic field strength (see, e.g., Aharonian et al. 2005). For $\mathrm{X}$-ray and TeV gamma-ray emission regions of sizes $R_{\mathrm{X}}$ and $R_{\gamma}$, respectively, with $\overline{E_{\mathrm{X}}}$ and $\overline{E_{\gamma}}$ being the corresponding mean energies of the photons in $\mathrm{keV}$ and $\mathrm{TeV}$ units, the magnetic field is $B_{\text {pwn }} \sim 160\left(R_{\mathrm{X}} / R_{\gamma}\right)^{2}\left(\overline{E_{\mathrm{X}}} / \overline{E_{\gamma}}\right) \mu \mathrm{G}$. For the observed $R_{\mathrm{X}} / R_{\gamma} \approx 0.5, \overline{E_{\mathrm{X}}}=5 \mathrm{keV}$, and $\overline{E_{\gamma}}=5 \mathrm{TeV}$, the corresponding average magnetic field is $\sim 40 \mu \mathrm{G}$. This is much higher than what is suggested by modeling (see below) and also much higher than what is seen in other such evolved systems.

Note that the ratio $R_{\mathrm{X}} / R_{\gamma} \approx 0.5$ is likely an underestimate and the ASCA data suggest that it can be a factor of 2-3 larger (see Figure 4). Indeed, in the $A S C A$ images some diffuse emission appears to be seen up to $40^{\prime}$ away from the pulsar (Slane et al. 2004). The true extent and the non-thermal nature of the faint X-ray emission can only be measured in deep observations with XMM-Newton. Similarly, the TeV size we quote is a lower limit since more the extended portions away from the pulsar are likely to be the fainter than our detection threshold. The estimate of the average magnetic field should thus be taken as a crude order-of-magnitude estimate.

Another estimate of the magnetic field can be made by assuming that diffusion is the dominant transport mechanism throughout the nebula. In the simplest case of cross-field diffusion (the Bohm limit), the diffusion constant is given by $D=\gamma m c^{3} / 3 e B$, where $\gamma$ is the electron Lorentz factor. Using the relation $E_{\gamma} \sim \gamma^{2} \epsilon$, where $E_{\gamma}$ is the mean upscattered IC photon energy and $\epsilon$ is the seed photon energy, the diffusion constant for electrons scattering on the CMB in a magnetic field $B_{-5}=B_{\text {pwn }} /\left(10^{-5} \mathrm{G}\right)$ can then be expressed as $D=8.5 \times 10^{25} E_{\gamma}^{1 / 2} B_{-5}^{-1} \mathrm{~cm}^{2} \mathrm{~s}^{-1}$. Assuming that particles travel during their characteristic cooling time of $\tau_{\gamma} \approx 100(1+$ $\left.14.4 B_{-5}^{2}\right)^{-1} E_{\gamma}^{-1 / 2} \mathrm{kyr}$ (see de Jager \& Djannati-Ataï 2009), the diffusion length is $\sim\left(6 D \tau_{\gamma}\right)^{1 / 2} \sim 13 B_{-5}^{-1 / 2}\left(1+14.4 B_{-5}^{2}\right)^{-1 / 2} \mathrm{pc}$ which translates into $\sim 20^{\prime} d_{1.4}^{-1}$ for $B_{\mathrm{pwn}}=5 \mu \mathrm{G}$. (Note that for a given distance to CTA 1 , this estimate depends only on the magnetic field strength.) This size roughly corresponds to the observed extent of the TeV source. Although small, such low magnetic field $(\sim 5 \mu \mathrm{G})$ was inferred through the multiwavelength spectral modeling for the Vela X plerion (de Jager et al. 2008). The low $B_{\text {pwn }}$ resulting from the Bohm diffusion estimates has been previously noticed for several other relic PWNe (e.g., de Jager \& Djannati-Ataï (2009) as well as by Uchiyama et al. (2009) and Anada et al. (2010) based on their analysis of the synchrotron spectra measured by Suzaku across the extent of TeV sources HESS J1825-137 and HESS J1809-193.)

Furthermore, we can use a dynamical model for the evolution of a PWN inside a non-radiative SNR (Gelfand et al. 2009) to estimate the physical properties of the PWN. The results are illustrated in Figure 6. We find that to correctly reproduce 


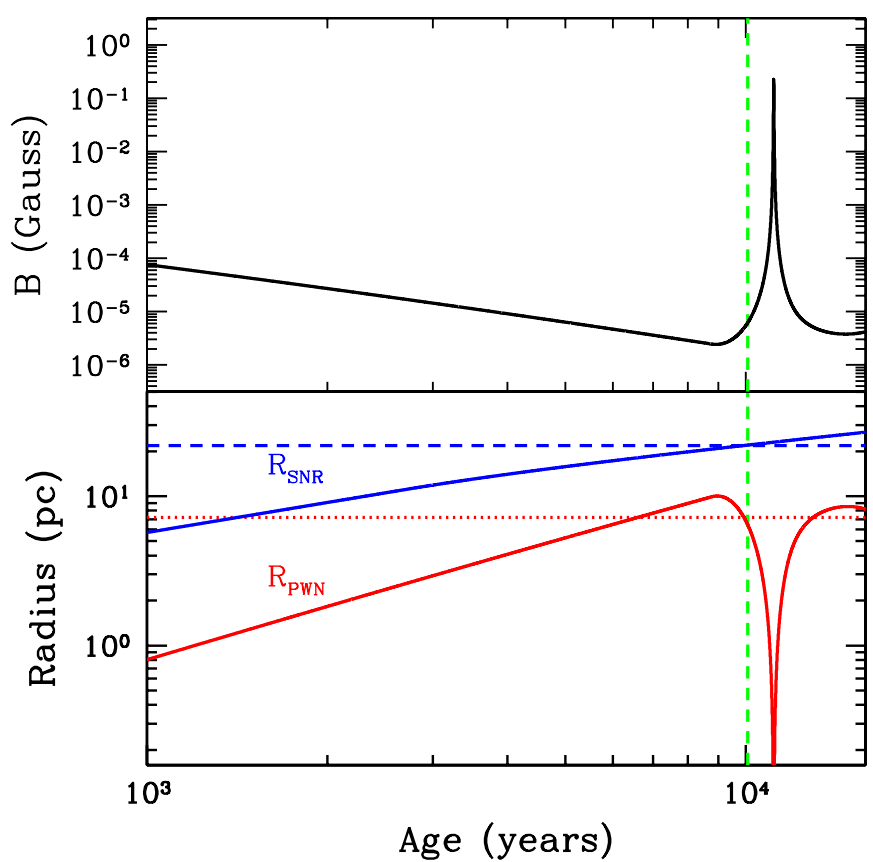

Figure 6. Upper: time evolution of the PWN magnetic field, using the model of Gelfand et al. (2009), with parameters given in Table 2. Lower: time evolution of the modeled SNR (blue) and PWN (red) radii. The horizontal dashed lines indicate the current values for CTA 1 . The vertical green line indicates the age at which the measured SNR radius is reached. (See the text for model description.) (A color version of this figure is available in the online journal.)

the radius of the SNR (lower panel, shown in blue) while simultaneously matching the estimated PWN radius (shown in red), the current spin-down power, and the total TeV flux, we require an ambient density $n_{0} \approx 0.07 \mathrm{~cm}^{-3}$. This is somewhat larger than that estimated from $A S C A$ measurements of the thermal X-ray emission (Slane et al. 1997, 2004), although those measurements were based on observations of a small fraction of the SNR shell.

Also shown in Figure 6 (upper panel) is the time evolution of the PWN magnetic field for this model. At the current age of $\sim 10 \mathrm{kyr}$ implied by the SNR radius, the PWN magnetic field strength is $\sim 6 \mu \mathrm{G}$. The recent decrease in the PWN radius and increase in the magnetic field result from the beginning of the SNR reverse shock interaction with the nebula, as suggested by other arguments presented above. Figure 7 shows the archival broadband data for CTA 1 along with the emission predicted for the model used in Figure 6 assuming a broken power-law injection of particles from the pulsar, for which a braking index of 3 is assumed. The model parameters are summarized in Table 2.

Radio observations of CTA 1 do not provide conclusive evidence for emission from the PWN (Pineault et al. 1997). We note that in their modeling, Zhang et al. (2009) assumed that the entire emission from the SNR was associated with the PWN. In fact, the PWN is much fainter. Here we have used the 1.4 GHz image from Pineault et al. (1997) to estimate the flux within a 20 arcmin radius around the pulsar, and have used this flux as an upper limit for the PWN emission. In Figure 7, we have extrapolated this upper limit to lower frequencies assuming a spectral index $\alpha=0.3$ (where $S_{v} \propto v^{-\alpha}$, is the flux at the frequency $\nu$ ) and to higher frequencies assuming $\alpha=0$. These index values represent the typical range observed in radio PWN, and the associated flux values correspond to conservative upper limits.

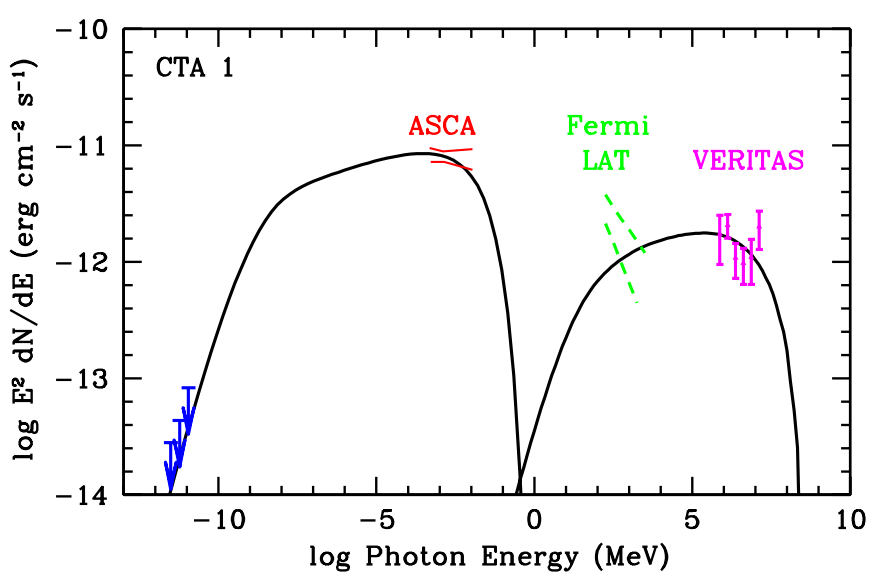

Figure 7. Broadband emission from CTA 1, along with the synchrotron and inverse Compton emission from the PWN predicted by the model of Gelfand et al. (2009) with parameters given in Table 2. See the text for the derivation of the $1.4 \mathrm{GHz}$ radio upper limits shown in blue. The red lines mark the errors on the X-ray spectrum measured by ASCA (Slane et al. 2004). The green, dashed lines mark the errors on the unpulsed Fermi-LAT spectrum (Abdo et al. 2012). (A color version of this figure is available in the online journal.)

Table 2

Model Parameters for Broadband Emission from CTA 1

\begin{tabular}{|c|c|}
\hline Parameter & Value \\
\hline \multicolumn{2}{|c|}{ Input } \\
\hline Explosion energy, $E_{S N}$ & $10^{51}$ erg (fixed) \\
\hline Ejecta mass, $M_{e j}$ & $6.1 M_{\odot}$ \\
\hline Ambient density, $n_{0}$ & $0.068 \mathrm{~cm}^{-3}$ \\
\hline Initial spin-down, $\dot{E}_{0}$ & $7.5 \times 10^{36} \mathrm{erg} \mathrm{s}^{-1}$ \\
\hline Spin-down timescale, $\tau_{0}$ & $3.2 \times 10^{3} \mathrm{yr}$ \\
\hline Braking index, $n$ & 3 (fixed) \\
\hline$\eta_{B}$ & 0.2 \\
\hline$\alpha_{1}$ & 0.5 \\
\hline$\alpha_{2}$ & 2.8 \\
\hline Break energy, $E_{b}$ & $50 \mathrm{GeV}$ \\
\hline \multicolumn{2}{|c|}{ Output } \\
\hline Age & $1.0 \times 10^{4} \mathrm{yr}$ \\
\hline$B_{\mathrm{PWN}}$ & $6.3 \mu \mathrm{G}$ \\
\hline$\dot{E}$ & $4.4 \times 10^{35} \mathrm{erg} \mathrm{s}^{-1}$ \\
\hline$\tau_{c}$ & $1.3 \times 10^{4} \mathrm{yr}$ \\
\hline$P_{0}$ & $155 \mathrm{~ms}$ \\
\hline
\end{tabular}

Note. See Gelfand et al. (2009) for parameter definitions.

The TeV spectrum predicted by Zhang et al. (2009) overpredicts the TeV flux and requires an initial spin period in the range 30-40 ms. In comparison, the model shown in Figure 7 agrees with the VERITAS data, within uncertainties, and we conclude $P_{0} \sim 155 \mathrm{~ms}$. This difference could arise from the fact that Zhang et al. (2009) assign the full radio flux from CTA 1 to the PWN, thus requiring significantly more energy in the electron spectrum. An additional difference in the models is that we have calculated the evolving magnetic field strength based on the fraction of spin-down energy injected as magnetic flux, whereas Zhang et al. (2009) assume a time-dependent field value that is independent of any other system parameters. Our results suggest a break energy of $\sim 50 \mathrm{GeV}$ with $\sim 80 \%$ of the spin-down power appearing in the form of particle flux. Like most such systems, CTA 1 is thus a particle-dominated PWN.

The dashed green curves in Figure 7 represent the best fit for the unpulsed Fermi-LAT spectrum published in Abdo et al. (2012). In the model calculation shown, the $\mathrm{TeV}$ emission is 


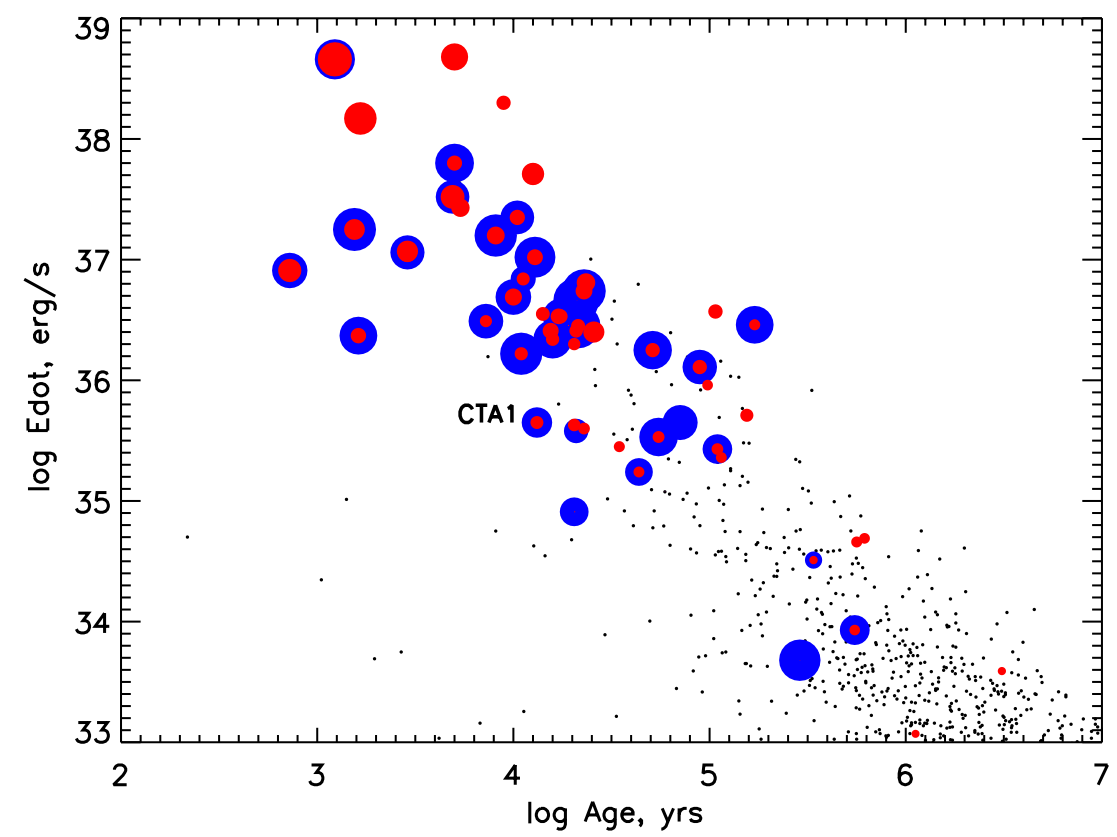

Figure 8. Pulsar spin-down luminosity vs. age, from Kargaltsev \& Pavlov (2010), with CTA 1 point indicated. Filled circles: X-ray (red) and TeV (blue) luminosities of PWNe or PWN candidates. Larger circle sizes correspond to higher luminosities in the corresponding waveband. The small black dots denote ATNF catalog pulsars (http://www.atnf.csiro.au/research/pulsar/psrcat; Manchester et al. 2005).

(A color version of this figure is available in the online journal.)

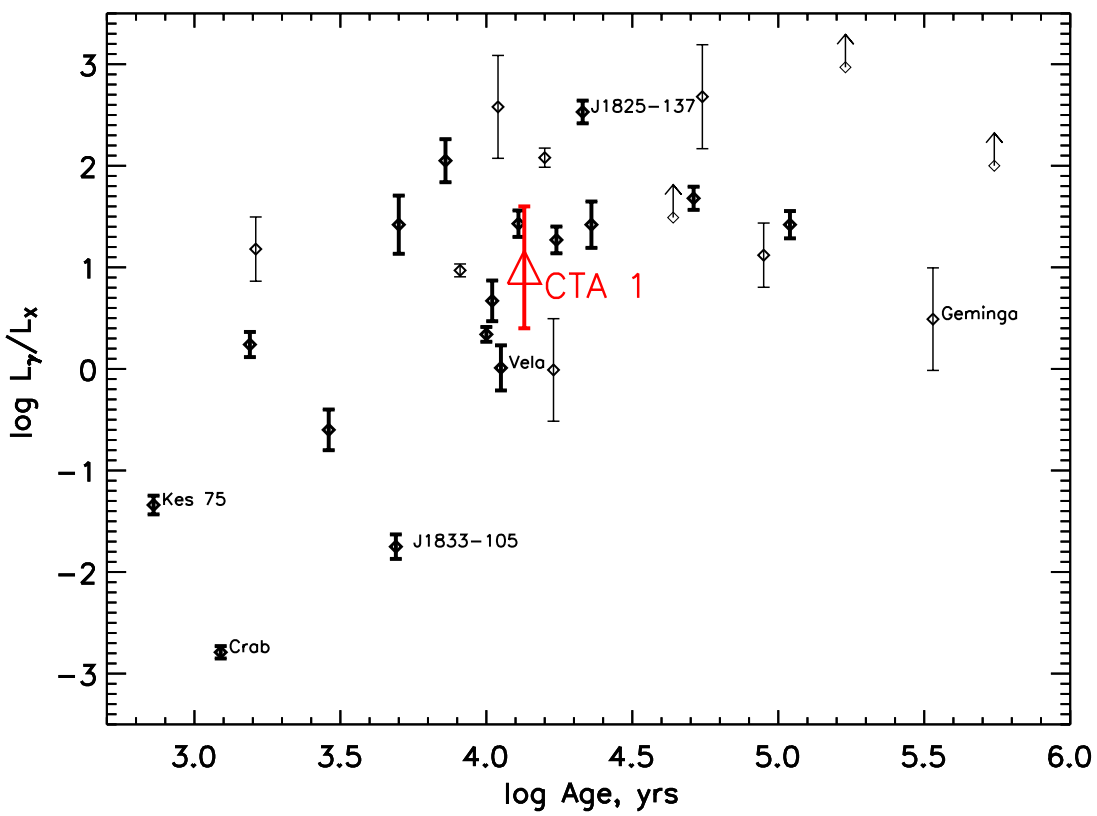

Figure 9. Plot of the ratio of TeV to X-ray luminosity vs. pulsar spin-down age, from Kargaltsev \& Pavlov (2010), with CTA 1 shown by the red triangle. The thick and thin error bars correspond to firm and tentative (or questionable) PWN associations, with selected objects labeled (see Kargaltsev \& Pavlov for further details).

(A color version of this figure is available in the online journal.)

produced by IC scattering of photons from the CMB, integrated starlight, and infrared emission from local dust, following the approximate prescription given by Strong et al. (2000). The model produces reasonable agreement with the radio, X-ray, and $\mathrm{TeV}$ data with a solution that gives approximately the correct pulsar spin-down power and characteristic age at the current epoch. However, the model is a poor fit to the reported Fermi-LAT spectrum. We have considered additional photon fields to produce enhanced IC emission at $\mathrm{GeV}$ energies, but have been unable to reproduce the published spectral index. We note that the reported unpulsed $\mathrm{GeV}$ emission is quite faint and it is in the presence of bright pulsed emission from PSR $\mathrm{J} 0007+7303$. It will be of considerable interest to carry out further investigations of this unpulsed emission as more FermiLAT data are accumulated.

\subsection{Comparison with Other Relic PWNe}

Figures 8 and 9 show the comparisons of the properties of CTA 1 with other PWNe and PWNe candidates detected at $\mathrm{TeV}$ energies. At the distance of $1.4 \mathrm{kpc}$, the $>1 \mathrm{TeV}$ luminosity of the PWN in CTA 1 is $9.4 \times 10^{32} \mathrm{erg} \mathrm{s}^{-1}$. Figure 8 
shows the relative luminosities of PWNe in the TeV and X-ray bands as functions of spin-down power and characteristic age (Kargaltsev \& Pavlov 2010). CTA 1 fits with the picture that TeV PWNe are generally found around pulsars with ages $\lesssim 100 \mathrm{kyr}$ and $\dot{E} \gtrsim 10^{35} \mathrm{erg} \mathrm{s}^{-1}$, although the $\mathrm{TeV}$ luminosities do not depend on the pulsar age nearly as much as the X-ray PWNe luminosities do. Figure 9 shows the distance-independent ratio of the $\mathrm{TeV}$ to X-ray luminosity as a function of characteristic age for a set of PWNe or PWN candidates, with CTA 1 marked by the red triangle. Additionally, the estimated size of the TeV emission region in CTA 1 is consistent with the sizes of $\mathrm{TeV}$ nebulae around pulsars with ages similar to that of PSR J0007+7303, although the large errors on the estimated distances prohibit a definite correlation. A comparison of CTA 1 with the TeV/X-ray PWN population supports the PWN origin of the TeV emission.

\section{SUMMARY AND CONCLUSIONS}

VERITAS has detected TeV gamma-ray emission coincident with SNR CTA 1. The emission is extended, with a centroid near the Fermi gamma-ray pulsar PSR J0007+7303 and its $\mathrm{X}$-ray PWN. The photon spectrum is well described by a power law with differential spectral index of $\Gamma=2.2 \pm 0.2_{\text {stat }} \pm 0.3_{\text {sys }}$ and an integral flux above $1 \mathrm{TeV}$ corresponding to $\sim 4 \%$ of the steady TeV gamma-ray emission from the Crab Nebula. It is unlikely that the TeV emission is due to interaction of the CTA 1 shell with a dense molecular cloud, given the lack of evidence for such a cloud at other wavelengths ( $60 \mu \mathrm{m}$ IR or H I maps). We have analyzed archival X-ray data from ROSAT $(0.5-2 \mathrm{keV})$ and $A S C A(4-10 \mathrm{keV})$ of the large-scale nebula and XMMNewton $(0.5-10 \mathrm{keV})$ and Chandra $(0.5-8 \mathrm{keV})$ of the region close to the pulsar and find that the large-scale emission seems to match the $\mathrm{TeV}$ morphology. The positional coincidence with the pulsar, small extent of the TeV emission, and X-ray/TeV luminosities strongly argue for a PWN origin. We have estimated the magnetic field strength assuming particle transport by either diffusion or by advection. A more detailed dynamical model of the SNR-PWN system suggests a $6 \mu \mathrm{G}$ field along with a recent interaction between the PWN and the SNR reverse shock.

This research is supported by grants from the U.S. Department of Energy Office of Science, the U.S. National Science Foundation and the Smithsonian Institution, by NSERC in Canada, by Science Foundation Ireland (SFI 10/RFP/AST2748) and by STFC in the U.K. We acknowledge the excellent work of the technical support staff at the Fred Lawrence Whipple Observatory and at the collaborating institutions in the construction and operation of the instrument.

O.K. was supported through the National Science Foundation grant No. AST0908733. P.S. acknowledges support from NASA contract NAS8-03060.

\section{REFERENCES}

Abdo, A. A., Ackermann, M., Ajello, M., et al. 2010, ApJ, 712, 1209 Abdo, A. A., Ackermann, M., Atwood, W. B., et al. 2008, Sci, 322, 1218 Abdo, A. A., Wood, K. S., DeCesar, M. E., et al. 2012, ApJ, 744, 146 Acciari, V. A., Beilicke, M., Blaylock, G., et al. 2008, ApJ, 679, 1427 Aharonian, F., Akhperjanian, A. G., Bazer-Bachi, A. R., et al. 2006, ApJ, 636, 777

Aharonian, F. A., Akhperjanian, A. G., Bazer-Bachi, A. R., et al. 2005, A\&A, 442, L25

Aharonian, F. A., Hofmann, W., Konopelko, A. K., \& Vöölk, H. J. 1997, APh, 6,343
Anada, T., Bamba, A., Ebisawa, K., \& Dotani, T. 2010, PASJ, 62, 179

Atoyan, A. M., \& Aharonian, F. A. 1996, MNRAS, 278, 525

Berge, D., Funk, S., \& Hinton, J. 2007, A\&A, 466, 1219

Brazier, K. T. S., Reimer, O., Kanbach, G., \& Carraminana, A. 1998, MNRAS, 295,819

Caraveo, P. A., De Luca, A., Marelli, M., et al. 2010, ApJL, 725, 6

Cogan, P. 2008, in Proc. 30th ICRC, Vol. 3, The VERITAS Gamma-ray Analysis Suite, ed. R. Caballero, J. C. D’Olivo, G. Medina-Tanco, L. Nellen, F. A. Sánchez, \& J. F. Valdeé-Galicia (Mexico City, Mexico: Universidad Nacional Autónoma de México), 1385

Daniel, M. K. 2008, in Proc. 30th ICRC, Vol. 3, The VERITAS Standard Data Analysis, ed. R. Caballero, J. C. D’Olivo, G. Medina-Tanco, L. Nellen, F. A. Sánchez, \& J. F. Valdeé-Galicia (Mexico City, Mexico: Universidad Nacional Autónoma de México), 1325

de Jager, O., Slane, P. O., \& LaMassa, S. 2008, ApJL, 689, 125

de Jager, O. C., \& Djannati-Ataï, A. 2009, in Neutron Stars and Pulsars, ed. W. Becker (Astrophysics and Space Science Library, Vol. 357; Berlin: Springer), 451

Fomin, V. P., Stepanian, A. A., Lamb, R. C., et al. 1994, APh, 2, 137

Gaensler, B. M., \& Slane, P. O. 2006, ARA\&A, 44, 17

Gelfand, J. D., Slane, P. O., \& Zhang, W. 2009, ApJ, 703, 2051

Hall, T. A., Wakely, S. P., \& the VERITAS Collaboration, 2001, in Proc. 27th ICRC, ed. W. Droege, G. Hainzelmann, K. H. Kampert et al. (Copernicus Gesellschaft: Katlenburg-Lindau, Germany)

Halpern, J. P., Camilo, C., \& Gotthelf, E. V. 2007, ApJ, 668, 1154

Halpern, J. P., Gotthelf, E. V., Camilo, F., Helfand, D. J., \& Ransom, S. M. 2004, ApJ, 612, 398

Halpern, J. P., \& Holt, S. S. 1992, Natur, 357, 222

Harris, D. E., \& Roberts, J. A. 1960, PASP, 72, 347

Hartman, R. C., Bertsch, D. L., Bloom, S. D., et al. 1999, ApJS, 123, 79

Hillas, M. 1985, in Proc. 19th ICRC, ed. F. C. Jones, J. Adams, \& G. M. Mason (Hanover, MD: NASA STI), 445

Hinton, J. A., \& Hofmann, W. 2010, ARA\&A, 47, 523

Holder, J., Aliu, E., Arlen, T., et al. 2011, in Proc. 32nd ICRC, Beijing (arXiv:1111.1225v1)

Kargaltsev, O., \& Pavlov, G. G. 2008, in AIP Conf. Proc., 983, 40 Years of Pulsars: Millisecond Pulsars, Magnetars and More, ed. C. Bassa, Z. Wang, A. Cumming, \& V. M. Kaspi (Melville, NY: AIP), 171

Kargaltsev, O., \& Pavlov, G. G. 2010, in AIP Conf. Proc., 1248, X-ray Astronomy 2009; Present Status, Multi-wavelength Approach and Future Perspectives, ed. A. Comastri, L. Angelini, \& M. Cappi (Melville, NY: AIP), 25

Kennel, C. F., \& Coroniti, F. V. 1984, ApJ, 283, 694

Khelifi, B., Barrau, A., Bazer-Bachi, R., et al. 2001, in Proc. 27th ICRC, ed. W. Droege, G. Hainzelmann, K. H. Kampert et al. (Copernicus Gesellschaft: Katlenburg-Lindau, Germany)

Komin, N., \& the HESS Collaboration, 2011, in Proc. 23rd Rencontres de Blois (arXiv:1201.0641)

Krawczynski, H., Carter-Lewis, D. A., Duke, C., et al. 2006, APh, 25, 380

LaMassa, S. M., Slane, P. O., \& de Jager, O. C. 2008, ApJL, 689, 121

Li, T.-P., \& Ma, Y.-Q. 1983, ApJ, 272, 317

Manchester, R. N., Hobbs, G. B., Teoh, A., \& Hobbs, M. 2005, AJ, 129, 1993

Montmerle, T. 1979, ApJ, 231, 95

Ong, R. A., Acciari, V. A., Arlen, T., et al. 2009, in Proc. 31st ICRC, ed. J. Szabelski \& M. Giller (Lodz, Poland: Univ. Lodz) (arXiv:0912.5355)

Pavlov, G. G., Teter, M. A., Kargaltsev, O., \& Sanwal, D. 2003, ApJ, 591, 1157

Pineault, S., Landecker, T. L., Madore, B., \& Gaumont-Guay, S. 1993, AJ, 105,1060

Pineault, S., Landecker, T. L., Swerdlyk, C. M., \& Reich, W. 1997, A\&A, 324, 1152

Roberts, M. S. E., Romani, R. W., \& Kawai, N. 2001, ApJ, 133, 451

Rowell, G., \& the HEGRA Collaboration, 2003, in Proc. 28th ICRC, ed. T. Kajita, Y. Asaoka, A. Kawachi, Y. Mastubara, \& M. Sasaki (Tokyo, Japan: Universal Academy Press, Inc.)

Seward, F. D., Schmidt, B., \& Slane, P. 1995, ApJ, 453, 284

Sieber, W., Salter, C. J., \& Mayer, C. J. 1981, A\&A, 103, 393

Slane, P., Seward, F. D., Bandiera, R., et al. 1997, ApJ, 485, 221

Slane, P., Zimmerman, E. R., Hughes, J. P., et al. 2004, ApJ, 601, 1045

Strong, A. W., Moskalenko, I. V., \& Reimer, O. 2000, ApJ, 537, 763

Temim, T., Slane, P., Gaensler, B. M., Hughes, J. P., \& Van Der Swaluw, E. 2009, ApJ, 691, 895

Torres, D. F., Romero, G. E., Dame, T. M., et al. 2003, PhR, 382, 303 Uchiyama, H., Matsumoto, H., Tsuru, T. G., et al. 2009, PASJ, 61, S189 Weekes, T. C., Cawley, M. F., Fegan, D. J., et al. 1989, ApJ, 342, 379

Zhang, L., Jiang, Z. J., \& Jin, G. F. 2009, ApJ, 699, 507 